\title{
mRNA Profiling of a Well-differentiated G1 Pancreatic NET Correlates with Immunohistochemistry Profile
}

\section{Abhirami Venugopal}

Deakin University - Melbourne Burwood Campus

Jessie Gillick-Walker

Deakin University - Melbourne Burwood Campus

Agnes A Michalczyk

Deakin University - Melbourne Burwood Campus

\section{Mustafa Khasraw}

Duke University Biology-Forestry Library: Duke University

M Leigh Ackland ( $\square$ leigha@deakin.edu.au)

Deakin University - Melbourne Burwood Campus https://orcid.org/0000-0002-7474-6556

Research article

Keywords: Neuroendocrine Tumours (NET), biomarkers, mRNA, qRT-PCR, immunohistochemistry

Posted Date: November 5th, 2020

DOl: https://doi.org/10.21203/rs.3.rs-101295/v1

License: (c) (1) This work is licensed under a Creative Commons Attribution 4.0 International License. Read Full License 


\section{Abstract}

Background: Neuroendocrine neoplasms (NENs) are a complex group of tumours that occur in many organs. The routinely used IHC markers for NEN diagnosis include CgA, synaptophysin, Ki67 and CD56. These have limitations, including lack of correlation to clinical outcomes and their presence in nontumour tissue. Identification of additional markers and more quantitative analyses of tumour tissue has the potential to contribute to improved clinical outcomes. We used qRT-PCR to profile the expression levels of a panel of markers in tumour and matched non-tumour tissue from a G1 pancreatic neuroendocrine tumour. Differences in mRNA levels between tumour and non-tumour tissue were compared with IHC analyses of the same sample.

Methods: Twenty-seven markers associated with tumour characteristics including neuroendocrine status, proliferation, stem cell phenotype, angiogenesis, epithelial to mesenchymal transition, cell adhesion, differentiation and tumour suppression were selected from previous studies. mRNA levels of these markers were measured in tumour and non-tumour in a G1 pancreatic tissue sample using qRT-PCR. IHC was carried out on the same tissue to detect the corresponding marker proteins.

Results: Of the twenty seven markers analysed, seven showed higher mRNA levels in tumour relative to non-tumour while thirteen markers had lower expression in tumour relative to non-tumour tissue. The most substantial difference in mRNA levels were a gain of CgA, CD56, b-catenin, CK20, PDX1 and p53 and loss of Ki67, PCAD, CK7, CD31, MENA, ECAD, EPCAM, CDX2 and CK6. Comparison of qRT-PCR data with IHC showed correlation between fifteen markers.

Conclusions: qRT-PCR is a relatively quantitative diagnostic tool with the advantage of being reproducible, fast, cheap and accurate. Differential marker profiling using qRT-PCR has the potential to improve the defining of tumour phenotypes and, in combination with IHC may have clinical utility towards improving stratification or distinguishing tumour grades. The inclusion of matched controls enables a more comparative analysis in comparison to many previous studies that report tumour data only. The results need to be validated in a cohort of different grades of NENs and related to clinical outcomes.

\section{Background}

Neuroendocrine neoplasms (NENs) can occur in all organs of the body, with the gastrointestinal system being a common site of localisation. Some features of neuroendocrine differentiation are common to all NENs while others may be site-specific [1]. This has caused confusion in classification of NENs because morphologically similar tumours can be found in different organs.

Histological morphology and immunohistochemical criteria are routinely used to diagnose NENs. Established diagnostic immunohistochemical markers include Ki-67 a marker of proliferation, chromogranin A ( $\mathrm{CgA})$, synaptophysin, and CD56 [2, 3]. Additional markers including CDX2, a transcription factor required for intestinal differentiation [4, 5], cytokerations, [6] and the transcription 
factor PDX-1 that is required for pancreatic ductal and islet cell development [7]. It is clear that the complexity of a tumour cannot be defined by a small set of markers but on the other hand there is a limit to the number of tests that can be carried out for routine diagnostic purposes.

Based on the 2019 WHO classification [8], NENs are classified into three grades: Grade 1 (low grade) with a mitotic rate $<2$ and $\mathrm{Ki}-67<3 \%$ (NET, G1), Grade 2 (intermediate) with a mitotic rate $<2$ and $\mathrm{Ki}-673-20 \%$, (NET, G2) and Grade 3 (high) with both mitotic rates and Ki-67 > 20\% (NET, G3). NECs are small cell type or large cell type, both poorly differentiated and with both mitotic rate and Ki-67 > 20\%.

In a study of 200 patients with pancreatic endocrine carcinoma, Ki-67 index was the major risk factor in relation to disease progression [9]. While Ki67 index is a WHO standard indicator of tumour proliferation and predictor of disease outcome, significant inter and intra-lab variability in Ki-67 grading due to technical variations and observer differences has been demonstrated [10]. The 2010 International Ki67 in Breast Cancer Working Group acknowledged the enormous variation in analytical practice that limits the value of Ki67 [11].

A lack of reliable predictive and prognostic markers in neuroendocrine neoplasms on which to base therapeutic choices was identified by the ENETs $[12,13]$. Single markers cannot define the numerous cellular changes associated with cancer. Analysis of additional molecules whose function is known to be altered in cancer including markers of proliferation, metabolic activity, invasive potential, metastatic propensity would provide possibilities for stratification of tumours that may inform treatment.

The aims of this study were to use RT-qPCR to measure mRNA levels of twenty seven markers in a pancreatic G1 NET tumour relative to matched non-tumour tissue, to obtain a marker profile that could have clinical utility towards improving stratification of G1 pancreatic tumours or distinguishing tumour grades.

\section{Case Report:}

A 66 year-old gentleman presented to his chiropractor with lower back pain having been asymptomatic for the previous 6 months. An ultrasound scan of his kidneys and a subsequent CT KUB + Abdo/Pelvis showed two solid lesions at the mid pole and lower pole of the right kidney and one heterogeneous solid lesion at the lower pole of the left kidney. It also showed a lesion in the pancreatic head measuring $3 \mathrm{~cm}$ with a dilated pancreatic duct and two small lymph nodes in the periportal region.

A CT scan of the chest was done to complete staging and this showed a $3 \mathrm{~mm}$ nodule in the inferior segment of the lingula. This was indeterminate and too small to be characterized. Apart from that, his CT of the abdomen and pelvis was reviewed and this confirmed two solid lesions in the right kidney and also one in the left kidney. The pancreatic head lesion was also identified with the two small lymph nodes in the peri-portal region. In terms of blood tests, his tumour markers were all within the normal limits. This included a CA19.9 of 32, CEA 1.8, PSA $<0.1$ and alpha feta protein of 4 . 
Histopathological analysis identified a well differentiated pancreatic endocrine neoplasm, low grade which was encapsulated on the external surfaces. However, elsewhere within the pancreas, tumour had infiltrated into the adjacent exocrine gland. The tumour had an organoid growth pattern with tubular, alveolar and trabecular groupings separated by a fibrous stroma. Cells showed mild nuclear pleomorphism with small nucleoli and gritty chromatin with abundant granular cytoplasm. Mitoses were not seen in $50 \mathrm{HPF}$ and no necrosis was present. Ki-67 staining was variable with generally less than $2 \%$ staining positive. No perineural or lymphovascular space invasion was identified. The resections margins were free of tumour with $15 \mathrm{~mm}$ of clearance at the proximal pancreatic margin and not present on the inked external surface which was separated from the tumour by $1 \mathrm{~mm}$ thick fibrous capsule. A diagnosis of well differentiated pancreatic endocrine neoplasm, low grade pT2NX, was made.

\section{Methods}

\section{Sample Collection:}

The pancreatic tumour with its matched adjacent non-tumour tissue was obtained from the Victorian Cancer Biobank (VCB) and stored at $-80^{\circ} \mathrm{C}$ until use. Regions of patient tissue showing normal pancreatic morphology were classed as non-tumour and regions where tissue was disorganised, containing cells with irregular nuclei and heterogeneity of size were classified as tumour tissue [1].

\section{Ethics:}

The experimental protocol received ethics approval from Deakin University (2014-239).

Quantitative Real-Time Reverse Transcription PCR (qRT-PCR)

\section{RNA Extraction:}

A $2 \mathrm{~mm} \times 2 \mathrm{~mm}$ frozen tissue was cut and ground using mortar and pestle in the presence of liquid nitrogen and further homogenised by passing through 20 gauge needle and syringe multiple times. RNA was extracted from the tissue samples using the RNeasy mini kit (Qiagen) as per manufacturers instruction. RNA concentrations were measured using the Thermo Scientific NanoDrop 1000. Only RNA that passed the QC of 260/280 ratio of 2 was reverse transcribed into cDNA.

\section{Reverse Transcription:}

RNA was converted into cDNA using Applied Biosystems High-Capacity cDNA Reverse Transcription Kit as per manufacturer's instruction. A working solution of $5 \mathrm{ng} / \mathrm{ul}$ was prepared by diluting the stock using nuclease free water. 


\section{Real-Time PCR:}

10ul of PowerUp SYBR Green Master Mix (Applied Biosystems), 5ng of cDNA template, 250nM of each primer was made up to $20 \mathrm{ul}$ in a PCR reaction/well using nuclease free water. Template (20ng) was used for markers which were expressed in very low levels. GAPDH and b-actin were used as the endogenous control. Every run also included negative controls of master mix without cDNA to rule out for contamination. The PCR cycle was $50^{\circ} \mathrm{C}-2 \mathrm{~min}, 95^{\circ} \mathrm{C}-10 \mathrm{~min}$ and 45 cycles of $95^{\circ} \mathrm{C}-15 \mathrm{~s}, 60^{\circ} \mathrm{C}-1 \mathrm{~min}$ and a final extension for $95{ }^{\circ} \mathrm{C}-15 \mathrm{~s}$. The plate was loaded on to Biorad CFX96 and the marker levels were analysed using relative quantification method (DDCt). All markers were tested for 3 individual qRT-PCR runs and triplicate experiments were run for each sample. The fold change was calculated on excel and two tailed $T$ test was used to determine the statistical significance.

\section{Statistics:}

\section{T-test:}

Two-tailed t-test with unequal variance was used to find the probability value ( $p$-value) between each normal and tumour sample and its positive and negative standard deviation values. P-values less than 0.05 were concluded to be statistically significant.

\section{One-way ANOVA:}

For tissue specific marker profiling, expression levels of all markers in each of normal and tumour tissue was calculated using expression of DAXX as control. One-way ANOVA with post hoc tukey test was used to study the significance of the markers in each profile. Markers with a $p$ value $<0.05$ in comparison to DAXX expression was identified to be significantly different in expression.

\section{Immunohistochemistry (IHC):}

Tissue Sectioning: A 3mm X 3mm tissue sample cut and a block prepared using OCT then frozen in liquid nitrogen. Sections of 5-8um thickness were sectioned and collected on a charged slide using a Thermo Scientific HM525NX cryostat. The slides were air dried and stored in $-80^{\circ} \mathrm{C}$ until use.

\section{Immunostaining:}

Frozen sections were rinsed three times with 1XPBS for 10 minutes then fixed in $4 \%$ paraformaldehyde (PFA). Slides were washed three times with $1 X$ PBS then incubated in $0.3 \%$ hydrogen peroxide for $10 \mathrm{~min}$ followed by 10 min incubation with $0.1 \%$ triton X with three 1 X PBS washes between the two incubations. 
The sections were blocked in $2 \%$ BSA for 45 min then incubated with primary antibodies at $4^{\circ} \mathrm{C}$ overnight. The following day the slides were washed using 1X PBS and incubated with horse radish peroxidase or alkaline phosphatase enzyme tagged secondary antibody for 30 minutes. The corresponding substrate, ImmPACT DAB or alkaline phosphatase (Vector Laboratories) was then added, followed by counterstaining with methyl green or haematoxylin and mounting using DPX solution (Sigma Aldrich, Australia).

\section{Imaging:}

Images were taken at 40X, 100X and 400X magnifications using an Olympus BX43 light microscope fitted with a DP21 camera. Images were collected and analysed by comparing the intensity of label in the tumour sample in comparison to that seen in the matched normal tissue.

\section{Results}

\section{Selection of markers:}

Twenty-seven markers previously associated with a range of carcinoma characteristics with purported preventive or prognostic value were selected for the current study. Table 1 lists these markers and their characteristics including neuroendocrine-specific markers and those that are associated with a cancer phenotype including markers for proliferation, stem phenotype, angiogenesis, EMT, cell adhesion, differentiation and tumour suppression. 
Table 1

List of markers and associated characteristics

\begin{tabular}{|ll|}
\hline Categories & Markers \\
\hline Neuroendocrine & $\begin{array}{l}\text { Chromogranin A (CgA), synaptophysin, neuron specific enolase } \\
(\text { NSE), CD56 }\end{array}$ \\
\hline Proliferation & Ki-67, PCNA, DAXX \\
\hline Stem Cell & CD24, CD44, CD49 (Integrin alpha-6) \\
\hline Angiogenesis & CD31, CD45 \\
\hline $\begin{array}{l}\text { Epithelial to } \\
\text { Mesenchymal }\end{array}$ & Vimentin, $\beta$-catenin, MENA \\
\hline Transition (EMT) & \\
\hline Cell Adhesion & Laminin, E-cadherin (ECAD), P-cadherin (PCAD), EpCAM, $\beta$ - $\beta$-catenin, CD56, \\
\hline Differentiation & CDX2, PDX1, CD14, CK6, CK7, CK13, CK20 \\
\hline Tumour suppression & P53 \\
\hline
\end{tabular}

\section{mRNA levels of markers in NEC and matched control tissue:}

mRNA transcript levels of each marker were measured in tumour relative to matched non-tumour tissue. The relative expression of markers was first expressed as fold change calculated using $\beta$-actin as the endogenous control. Tumour marker mRNA levels were then expressed relative to the control sample, the non-tumour tissue with a mRNA expression value of one. Of the twenty seven markers, mRNA levels of twenty were significantly different in tumour compared to the non-tumour (Fig. 1A). Higher expression levels in tumour relative to non-tumour tissue of $>10$ fold were found for $\mathrm{CgA}, 2-10$ fold higher mRNA levels were found for CD56, $\beta$-catenin, PDX1, CK20, and P53 and 1-2 fold higher mRNA levels was found in CD45 tumour tissue compared with the non-tumour tissue. mRNA levels of Ki-67, CD24, CD44, CD31, MENA, CD49, ECAD, PCAD, EpCAM, CDX2, CK6, CK7, CK13, were significantly decreased in tumour tissue relative to non-tumour tissue. NSE and vimentin was higher in tumour relative to non-tumour tissue, but not significantly. The markers synaptophysin, PCNA, DAXX, laminin and CD14 had similar expression levels in tumour and non-tumour tissue. Table 2 summarises the mRNA transcript levels of each marker in tumour tissue relative to non-tumour tissue. 
Table 2

qPCR analysis of the expression levels of 27 markers in tumour tissue compared to non-tumour, using $\beta$ actin as endogenous control.

\begin{tabular}{|c|c|}
\hline Marker & Expression level \\
\hline $\mathrm{CgA}$ & $+++^{\star}$ \\
\hline synaptophysin & $=$ \\
\hline NSE & + \\
\hline CD56 & $++*$ \\
\hline Ki-67 & --* \\
\hline PCNA & $=$ \\
\hline DAXX & $=$ \\
\hline CD24 & $-\star$ \\
\hline CD44 & $-\star$ \\
\hline CD31 & $-*$ \\
\hline CD45 & $+^{*}$ \\
\hline Vimentin & + \\
\hline$\beta$-catenin & $++*$ \\
\hline MENA & -* \\
\hline CD49 & $-\star$ \\
\hline Laminin & $=$ \\
\hline ECAD & -* \\
\hline PCAD & $--^{\star}$ \\
\hline EpCAM & $-\star$ \\
\hline $\mathrm{CDX} 2$ & $-\star$ \\
\hline PDX1 & $++^{\star}$ \\
\hline CD14 & $=$ \\
\hline
\end{tabular}

\footnotetext{
+ and - indicates whether marker mRNA transcript levels are higher or lower in the tumour tissue compared to the matched no-tumour tissue. * represents significant changes $(p<0.05)$.

$\therefore$ 1-2 fold change lower compared to normal; - -: 2-10 fold change lower compared to normal; - - - : $>10$ fold change lower compared to normal; $+: 1-2$ fold change higher relative to normal; $++: 2-10$ fold change higher relative to normal; +++:>10 fold change relative to normal; =: similar in tumour and nontumour
} 


\begin{tabular}{|c|c|}
\hline Marker & Expression level \\
\hline CK6 & $-\star$ \\
\hline CK7 & $-{ }^{\star}$ \\
\hline CK13 & $-\star$ \\
\hline CK20 & $++^{*}$ \\
\hline P53 & $++^{*}$ \\
\hline \multicolumn{2}{|c|}{$\begin{array}{l}+ \text { and }- \text { indicates whether marker mRNA transcript levels are higher or lower in the tumour tissue } \\
\text { compared to the matched no-tumour tissue. * represents significant changes }(p<0.05) \text {. }\end{array}$} \\
\hline \multicolumn{2}{|c|}{$\begin{array}{l}-: 1-2 \text { fold change lower compared to normal; }--: 2-10 \text { fold change lower compared to normal; }---: \\
>10 \text { fold change lower compared to normal; }+1-2 \text { fold change higher relative to normal; }++: 2-10 \text { fold } \\
\text { change higher relative to normal; +++:>10 fold change relative to normal; =: similar in tumour and non- } \\
\text { tumour }\end{array}$} \\
\hline
\end{tabular}

The mRNA levels of markers were compared relative to each other for both tumour and non-tumour tissue. This provided a tissue-specific marker profile for tumour and non-tumour tissue. DAXX was selected for standardisation of mRNA levels as its expression was similar in tumour and non-tumour tissue. DAXX was assigned a value of 1 . The relative expression level of each marker in non-tumour tissue relative to DAXX is shown in Fig. 1B. CD24, CD44, CD31, EpCAM and CK6 were expressed significantly higher than DAXX in non-tumour tissue while CgA, synaptophysin, CD56, Ki-67, PCNA, CD45, vimentin, $\beta$-catenin, MENA, laminin, ECAD, PCAD, PDX1, CD14, CK7, CK20 and P53 had significantly lower expression levels relative to DAXX in non-tumour tissue. In tumour tissue, CgA and NSE mRNA levels were significantly greater than DAXX while synaptophysin, CD56, Ki67, PCNA, CD44, CD31, CD45, vimentin, $\beta$ catenin, MENA, CD49, laminin, ECAD, PCAD, EPCAM, CDX2, PDX1, CD14, CK7, CK13, CK20 and p53 had mRNA levels that were significantly lower than DAXX (Fig. 1C).

\section{Immunohistochemical analysis of tumour and matched control tissue:}

Immunohistochemical analysis was carried out for each of the twenty seven markers in tumour and nontumour tissue. Images of one representative marker from each category of tumour markers including neuroendocrine origin, cell proliferation, stem cell phenotype and angiogenesis is shown in Fig. 2 and one representative marker from each category of EMT, cell adhesion, differentiation and tumour suppression is illustrated in Fig. 3. The IHC data is summarised in Table 3. 
Table 3

Immunohistochemistry analysis of the expression levels of 27 markers in tumour tissue compared to non-tumor.

\begin{tabular}{|c|c|c|}
\hline Marker & Expression in Non-Tumour Tissue & Expression in Tumour Tissue \\
\hline $\mathrm{CgA}$ & - & +++ \\
\hline synaptophysin & - & - \\
\hline NSE & - & +++ \\
\hline CD56 & + & ++ \\
\hline Ki-67 & - & + \\
\hline PCNA & - & + \\
\hline DAXX & - & +++ \\
\hline CD24 & - & +++ \\
\hline CD44 & + & +++ \\
\hline CD31 & - & + \\
\hline CD45 & - & +++ \\
\hline Vimentin & - & ++ \\
\hline$\beta$-catenin & - & +++ \\
\hline MENA & + & + \\
\hline CD49 & + & + \\
\hline Laminin & +++ & + \\
\hline ECAD & + & ++ \\
\hline PCAD & - & - \\
\hline ЕрСАM & + & + \\
\hline CDX2 & - & - \\
\hline PDX1 & ++ & +++ \\
\hline CD14 & ++ & + \\
\hline CK6 & ++ & + \\
\hline
\end{tabular}

* represents a similar expression pattern as obtained with mRNA analysis. + and - represent the increase and decrease in expression of marker in tumour tissue in comparison to the normal sample.

$\therefore$ Absence of marker expression; +:1-25\% field positive for marker expression; ++: $25-50 \%$ field positive for marker expression; +++: $>50 \%$ field positive for marker expression 


\begin{tabular}{|lll|}
\hline Marker & Expression in Non-Tumour Tissue & Expression in Tumour Tissue \\
\hline CK7 & ++ & - \\
\hline CK13 & ++ & - \\
\hline CK20 & - & ++ \\
\hline P53 & - & ++ \\
\hline $\begin{array}{l}\text { * represents a similar expression pattern as obtained with mRNA analysis. }+ \text { and }- \text { represent the } \\
\text { increase and decrease in expression of marker in tumour tissue in comparison to the normal sample. } \\
\text {-: Absence of marker expression; }+: 1-25 \% \text { field positive for marker expression; }++: 25-50 \% \text { field } \\
\text { positive for marker expression; }+++: ~>50 \% \text { field positive for marker expression }\end{array}$ \\
\hline
\end{tabular}

$\mathrm{CgA}$ staining was absent in non-tumour tissue but many cells in the tumour tissue were stained (Fig. 2A,B). Synaptophysin staining was absent in both non-tumour and tumour tissue. NSE showed no staining in non-tumour tissue and strong staining in most cells in tumour tissue. CD 56 showed $<10 \%$ stained cells in non-tumour tissue and staining of $50 \%$ of tumour tissue cells. Markers of proliferation Ki67 and PCNA (Fig. 2C,D) was absent in non-tumour cells and strong nuclear staining was observed in few cells in the tumour tissue. DAXX staining was absent in non-tumour tissue and present in most cells of the tumour tissue.

The stem cell marker, CD24 showed no staining in non-tumour tissue and strong staining in all cells of the tumour tissue (Fig. 2E,F), while CD44 showed $<10 \%$ stained cells in non-tumour tissue and strong staining in most cells of the tumour tissue. The markers of angiogenesis CD31 (Fig. 2G,H) and CD45 were not detected in non-tumour tissue but showed focal staining associated with blood vessels. Cells in nontumour tissue were non stained for the EMT markers vimentin and $\beta$-catenin (Fig. 3A,B) while MENA was found in a small proportion of cells in non-tumour tissue, but within the tumour tissue approximately $50 \%$ of cells were positive for vimentin and $\beta$-catenin, and had patchy staining for MENA.

Staining for the cell adhesion marker CD49 showed $10 \%$ positive cells in non-tumour tissue with less staining in tumour tissue. Laminin was strongly stained in the extracellular matrix in non-tumour tissue and in the tumour tissue the label was associated with blood vessels (Fig. 3C,D). ECAD staining was detected in $<25 \%$ of the cells in non-tumour tissue while the tumour tissue had $25-50 \%$ positive staining. PCAD was negative in both normal and tumour. EpCAM staining was found in $50 \%$ of cells in both nontumour and tumour tissue.

No staining for the differentiation marker CDX2 was detected in either non-tumour or tumour tissue. PDX1 staining was detected in both non-tumour and tumour tissue. Strong staining for CD14 was detected in non-tumour tissue with less label in tumour tissue. CK6 stained approximately $40 \%$ of cells in non-tumour tissue and $20 \%$ of cells in tumour tissue. Both CK7 (Fig. 3E,F) and CK13 showed strong focal staining in non-tumour tissue with little or no staining in tumour tissue. CK20 staining was not detected in nontumour tissue but over $50 \%$ of cells in tumour tissue were stained. Staining for the tumour suppressor 
P53 (Fig. 3G,H) was not detected in non-tumour tissue but was present in most cells within the tumour tissue.

\section{Comparison of immunohistochemistry and qPCR results}

The differences in marker staining between tumour and non-tumour obtained from IHC were compared with mRNA marker expression levels from the qRT-PCR analysis of tumour and non-tumour tissue (Table 4). CgA, synaptophysin, NSE, CD56, CD45, vimentin, $\beta$-catenin, CD49, EpCAM, PDX1, CK6, CK7, CK13, CK20, P53 were consistent in both mRNA and protein levels between tumour and non-tumour tissue. Other markers including Ki67, PCNA, DAXX, CD24, CD44, CD31, MENA, Laminin, ECAD, PCAD and CDX2 and CK14 showed different trends in expression between tumour and non-tumour with qRT-PCR compared to IHC. CDX2 and PCAD showed decreased mRNA levels in tumour relative to non-tumour and absence of these proteins in normal and tumour tissue.

Table 4

Comparison of marker expression levels in non-tumour and tumour tissue for IHC and mRNA

\section{Markers whose PCR expression levels between non-tumour and tumour correlated with IHC}

$\mathrm{CgA}$

Synaptophysin

NSE

CD56

CD45

Vimentin

$\beta$-catenin

CD49

EpCAM

PDX1

CK6

CK7

CK13

CK20

P53

\section{Markers whose PCR expression between non- tumour and tumour did not correlate with IHC}

Ki67

PCNA

DAXX

CD24

CD44

CD31

MENA

Laminin

ECAD

PCAD

CDX2

CD14

mRNA levels of Ki67, CD24, CD44, CD31, MENA and ECAD were observed to be reduced in the tumour tissue relative to the normal, whereas the protein expression was increased in the tumour compared to 
normal tissue. mRNA levels of PCNA, DAXX, laminin and CD14 in the tumour was equal to the levels seen in the non-tumour tissue whereas protein expression was seen to be increases for PCNA and decreased for laminin and CD14 in the tumour tissue relative to the non-tumour tissue.

\section{Discussion}

Limitations in the current tumour classification and grading for NEN subtypes have highlighted the lack of predictive and prognostic markers [12]. The routinely used immunochemical markers CgA, synaptophysin and CD56 confirm a neuroendocrine diagnosis and the proliferation marker Ki67 is used a key indicator for the grading of NENs [8]. These markers have some limitations including their lack of correlation to clinical outcomes and their presence in non-tumour tissue. The routinely used markers represent only a few of the molecules that characterise the tumour phenotype. Thus, additional markers are required to more comprehensively define NEN subtypes.

In this study we used quantitative real-time reverse transcription PCR (qRT-PCR) to measure the mRNA levels of twenty seven cancer markers associated with proliferation, metabolic activity, invasive potential and metastasis, in tissue from a patient with a G1 pancreatic NET. Comparison of mRNA marker levels from tumour tissue with adjacent non-tumour tissue from the same patient provided insights into the changes associated with acquisition of the cancer phenotype. The inclusion of matched controls enabled a comparative analysis, in comparison to many previous studies reporting tumour data only, which has limited value by itself when the status of the non-tumour tissue is unknown. Of the twenty seven markers, $\mathrm{CgA}$ had a transcript abundance that was 80 times higher in tumour relative to non-tumour. CD56 and p53 were 6.5-fold more abundant in tumour relative to non-tumour tissue and $\beta$-catenin, PDX1 and CK20 were 2-3 times as abundant in tumour than non-tumour tissue. The profile of the tumour tissue indicated an overall reduction in mRNA levels of the analysed markers relative to non-tumour tissue. Ki67, PCAD and CK7 expression was reduced up to 10-fold in the tumour relative to non-tumour with 2-9-fold reductions in CD24, CD44, CD31, CD49 MENA, ECAD, EPCAM, CDX2, CK6 and CK13. This suggests an overall loss of activity of these genes in the tumour. The most distinctive features of the tumour tissue were a gain in CgA, CD56, p53, PDX1, CK20 and a loss of Ki67, PCAD and CK7.

The observed increases in mRNA transcripts of the mesenchymal markers vimentin and $\beta$-catenin, decreased transcripts of the cell adhesion markers E-cadherin and P-cadherin and loss of differentiation markers CDX2, CK6, CK7 and CK13 are hallmarks of transition to a mesenchymal phenotype tumour cells. The epithelial to mesenchymal transition is a process by which normal epithelial cells lose their cell:cell and cell:matirix adhesion and acquire the migratory and invasive characteristics of a cancer cell [14].

This study indicates that qRT-PCR can distinguish phenotypic differences between tumour and nontumour tissue. Advantages of qRT-PCR are that it is a semi-quantitative tool that generates numerical values for marker expression so comparisons can be made between different patients, as each marker is measured relative to a housekeeping gene. Other benefits are that it is quick and multiple markers can be 
tested in a single run. qRT-PCR is cheap, requiring only a few enzymes, buffers and primers. The same set of identical primers can be used in multiple labs thereby reducing variabilities between labs. A further advantage of qRT-PCR is that the RNA is extracted from the whole tissue and same extract used for all marker analyses. The extract is representative of the whole tissue rather than a portion of the tissue. PCR avoids the use of an antibody and the variability associated with antibodies to the same protein but from different sources.

qRT-PCR measures mRNA levels markers relative to a housekeeping gene or control gene. This is selected to be stably expressed under different experimental conditions and for its expression to remain constant in different tissues and not influenced by the presence of disease so the expression of other genes can be measured relative to it. $\beta$-actin and GAPDH are commonly used housekeeping genes as their expression has been found to remain constant under different conditions $[15,16]$. However, studies indicate that some housekeeping genes could be better than the others for a particular sample, as the expression of these and various other housekeeping genes can be different in different sample cohort. Hence, Hence, we tested the $\beta$-actin and GAPDH housekeeping genes

and selected the one with least variation. We found $\beta$-actin to have less variation between tumour and non-tumour, compared to GAPDH for the pancreatic NET sample. Therefore, $\beta$-actin was selected as the housekeeping gene for analysis and future experiments.

To provide a profile of the different markers expressed in tumour and non-tumour tissue, we measured mRNA levels of markers in each of these tissues relative to each other using DAXX as a reference as this gene was expressed in similar levels in tumour and non-tumour. This data showing the pattern of expression of different markers in tumour and non-tumour tissue provides a profile that may have application in defining individual patients. CgA for example is seen to be significantly low in the normal tissue profile while it has a massive increase in the tumour tissue. Future studies are required to validate these data using a larger number of patients and determine whether such profiles have potential for stratification of G1 tumours. A larger cohort may establish the possibility to Identify markers that are evidently different between normal and tumour to draw out a reference profile for different grades of NETs.

We compared qRT-PCR results with IHC. Fifteen of the twenty seven markers showed the same expression trends between tumour and non-tumour tissue and twelve showed differences in trends between tumour and non-tumour tissue. The most notable differences between the qRT-PCR and the IHC results were Ki67, MENA, ECAD and the cluster of differentiation markers CD24, CD44 and CD31. Our data showed Ki67 was higher in tumour relative to non-tumour using IHC but qRT-PCR analysis indicated Ki67 mRNA was reduced in tumour tissue relative to the non-tumour. Previous studies comparing Ki67 protein with Ki67 mRNA levels indicated variable correlations amongst different tumour samples [17]. Variability in Ki67 expression has been attributed to changes in Ki67 levels with different stages of the cell cycle [18]. It is possible that the low levels of Ki67 found in our study may correspond with regions of the tumour containing non-cycling cells, given that IHC only utilises a small proportion of the total tumour mass. qRT- 
PCR for Ki67 was found to be more accurate than IHC in a breast cancer study in predicting patient response [19]. ECAD expression associated with breast cancer metastasis is understood to be regulated by cell surface integrin a $3 \beta 1$. a3 $\beta 1$ inversely affects the mRNA expression level of ECAD and this is counter balanced by the protein expression [20].

Our study showed that for four of the seven CD markers (CD24, CD44, CD31, CD14) mRNA and protein data did not correlate. This is consistent with a previous study where concordance between gene and protein expression for a range of $\mathrm{CD}$ antigens in normal and prostrate tumour samples was poor to moderate (Pearson correlations ranged from 0 to 0.63 ), attributed to low levels of protein expression, sample preparation as well as the real biological differences between protein and mRNA expression [21]. CD24 functions in cell adhesion and signaling, where high expression is associated with increased proliferation and invasion in pancreatic, colorectal and lung cancer but decreased proliferation and invasion in breast cancer cells [22]. The lack of direct correlation between mRNA and protein levels may be related to the complex regulatory pathways between CD24 transcription and translation. CD31 is an endothelial marker used as an indicator of blood vessels. Its expression may relate to the amount of vascular tissue which can vary considerably depending on the microscopic fields selected for IHC.

Synaptophysin was not detected by IHC and was only barely detectable by qRT-PCR, showing a slight increase in tumour relative to non-tumour. The levels of this transcript may not have been sufficient to produce detectable levels of protein. IHC staining for synaptophysin varies between different NENs and was reported as positive for approximately $60 \%$ of gastrointestinal neuroendocrine carcinomas [23]. EPCAM mRNA levels were lower in the tumour tissue than non-tumour and the $\mathrm{IHC}$ label was also lower in tumour relative to non-tumour however both tumour and non-tumour were categorised as $1-25 \%$ stained and not sufficiently different to be distinguishable. CD49 showed less mRNA in tumour than non-tumour. This pattern was also observed in IHC where staining was slightly less in tumour than non-tumour but both tumour and non-tumour showed $<25 \%$ positive staining.

$\mathrm{IHC}$ is the traditional method for tumour diagnosis, essential for establishing tissue morphology and providing information for grading of tumour biopsies. IHC provides information about morphology of tissue that cannot be obtained with PCR. IHC is a qualitative and relatively time-consuming procedure in which the scoring technique may dependent on the scorer. Small changes of a few percentage in the Ki67 index can affect the grading of the tumour which itself may have an influence on the type of treatment regime chosen. While the new WHO NEN guidelines have improved the stringency of IHC methods [8], the heterogeneity of tissue, differences in preparation of tissue, differences in counting approaches (automated versus manual) as well as variations in the sensitivity and specificity of antibodies adversely affects the prognostic potential of IHC [24]. IHC requires further development to become an immunoassay, not simply a stain [25].

\section{Conclusion}


qRT-PCR analysis of a G1 pancreatic sample revealed differential expression of 20 markers between tumour and matched normal tissue, out of a total of 27 markers tested. That many markers were found to be differentially expressed between tumour and non-tumour indicates marker profiling as a potential, additional diagnostic tool for better defining and stratification of tumours. As this was a pilot study based on a single NET case, the utility of the approach to measure multiple markers requires validation in a cohort study of different NEN grades and correlation with clinical outcomes. A better understanding of tumour cell phenotypes and their cell biology will contribute to improved clinical outcomes for patients.

\section{Abbreviations}

NETs: Neuroendocrine tumours, NENs: Neuroendocrine neoplasm, NEC: Neuroendocrine carcinoma, IHC: Immunohistochemistry, qRT-PCR: Real-time quantitative PCR

CgA: Chromogranin A, NSE: Neuron Specific Enolase, CK: Cytokeratin, EMT: Epithelial to Mesenchymal Transition

\section{Declarations}

\section{Ethics approval and consent to participate}

The project had ethics approval from the Victorian Cancer Biobank. The individual gave written consent. All patient information was de-identified. This study also received ethics approval from Deakin University Ethics Committee (2014-239).

\section{Consent for publication}

The patient gave written consent for their personal and clinical details to be published. There are no identifying images.

\section{Competing interests}

MK reports consultant or advisory roles for AbbVie, Ipsen, Pfizer Roche, and Jackson Laboratory for Genomic Medicine; research funding from AbbVie, Bristol-Myers Squibb (BMS), and Specialized Therapeutics. The other authors declare no competing interests.

\section{Funding}

We are grateful for funding from the Bourne Foundation to support a part time salary for AM. 
We acknowledge support from the Grace King Postgraduate Research Graduate Scholarship for provision the stipend (living costs) for AV to undertake a PhD.

\section{Authors contribution}

All authors have read and approved the manuscript. AV completed the qPCR for newly added markers, all the IHC staining, analysis and writing of the manuscript. JG-W generated and analysed the qPCR data for the markers initially selected. AM provided intellectual and technical inputs to the experimental design and analysis. MK provided intellectual inputs on the clinical aspect of the manuscript. MLA provided intellectual input into the study, interpretation of results and prepared the manuscript.

\section{Availability of data and Materials}

The raw qRT-PCR data and immunohistochemistry images are available.

\section{Acknowledgements}

Biospecimens used in this research were purchased from the Victorian Cancer Biobank, Victoria, Australia with appropriate ethics approval. The Victorian Cancer Biobank is supported by the Victorian Government.

\section{Author details}

${ }^{1}$ Centre for Cellular and Molecular Biology, School of Life and Environmental Sciences, Deakin University, Burwood, Victoria, Australia 3125, ${ }^{2}$ Duke University Medical Center, Durham, NC 27710, USA

\section{References}

1. Klimstra D, Modlin I, Coppola D, Lloyd R, Suster S: The pathological classification of neuroendocrine tumours Pancreas 2010, 39:707-712.

2. Ferolla P, Faggiano A, Mansueto G, Avenia N, Cantelmi MG, Giovenali P, Del Basso De Caro ML, Milone F, Scarpelli G, Masone $S$ et al: The biological characterization of neuroendocrine tumors: the role of neuroendocrine markers. J Endocrinol Invest 2008, 31(3):277-286.

3. Khan MS, Kirkwood A, Tsigani T, Garcia-Hernandez J, Hartley JA, Caplin ME, Meyer T: Circulating tumor cells as prognostic markers in neuroendocrine tumors. J Clin Oncol 2013, 31(3):365-372.

4. Kyriakopoulos G, Mavroeidi V, Chatzellis E, Kaltsas GA, Alexandraki KI: Histopathological, immunohistochemical, genetic and molecular markers of neuroendocrine neoplasms. Ann Trans/ Med 2018, 6(12):252-252. 
5. Barbareschi M, Roldo C, Zamboni G, Capelli P, Cavazza A, Macri E, Cangi MG, Chilosi M, Doglioni C: CDX-2 homeobox gene product expression in neuroendocrine tumors: its role as a marker of intestinal neuroendocrine tumors. Am J Surg Pathol 2004, 28(9):1169-1176.

6. Kende Al, Carr NJ, Sobin LH: Expression of cytokeratins 7 and 20 in carcinomas of the gastrointestinal tract. Histopathology 2003, 42(2):137-140.

7. Yang MX, Coates RF, Ambaye A, Cortright V, Mitchell JM, Buskey AM, Zubarik R, Liu JG, Ades S, Barry MM: NKX2.2, PDX-1 and CDX-2 as potential biomarkers to differentiate well-differentiated neuroendocrine tumors. Biomark Res 2018, 6(1):15.

8. Nagtegaal ID, Odze RD, Klimstra D, Paradis V, Rugge M, Schirmacher P, Washington KM, Carneiro F, Cree IA, Board tWCoTE: The 2019 WHO classification of tumours of the digestive system. Histopathology 2020, 76(2):182-188.

9. Panzuto F, Boninsegna L, Fazio N, Campana D, Pia Brizzi M, Capurso G, Scarpa A, De Braud F, Dogliotti L, Tomassetti $P$ et al: Metastatic and locally advanced pancreatic endocrine carcinomas: analysis of factors associated with disease progression. J Clin Oncol 2011, 29(17):2372-2377.

10. Mengel M, von Wasielewski R, Wiese B, Rüdiger T, Müller-Hermelink HK, Kreipe H: Inter-laboratory and inter-observer reproducibility of immunohistochemical assessment of the Ki-67 labelling index in a large multi-centre trial. J Pathol 2002, 198(3):292-299.

11. Dowsett M, Nielsen TO, A'Hern R, Bartlett J, Coombes RC, Cuzick J, Ellis M, Henry NL, Hugh JC, Lively $\mathrm{T}$ et al: Assessment of Ki67 in breast cancer: recommendations from the International Ki67 in Breast Cancer working group. J Natl Cancer Inst 2011, 103(22):1656-1664.

12. de Herder WW, Capdevila J: Unmet Needs in the Field of Neuroendocrine Neoplasms of the Gastrointestinal Tract, Pancreas, and Respiratory System: Reports by the ENETS Group. Neuroendocrinology 2019, 108(1):5-6.

13. Sorbye H, Baudin E, Borbath I, Caplin M, Chen J, Cwikla JB, Frilling A, Grossman A, Kaltsas G, Scarpa A et al: Unmet Needs in High-Grade Gastroenteropancreatic Neuroendocrine Neoplasms (WHO G3). Neuroendocrinology 2019, 108(1):54-62.

14. Hugo H, Ackland ML, Blick T, Lawrence MG, Clements JA, Williams ED, Thompson EW: Epithelialmesenchymal and mesenchymal-epithelial transitions in carcinoma progression. J Cell Physiol 2007, 213(2):374-383.

15. Aerts JL, Gonzales MI, Topalian SL: Selection of appropriate control genes to assess expression of tumor antigens using real-time RT-PCR. BioTechniques 2004, 36(1):84-86, 88, 90-81.

16. Liu DW, Chen ST, Liu HP: Choice of endogenous control for gene expression in nonsmall cell lung cancer. Eur Respir J 2005, 26(6):1002-1008.

17. Muftah AA, Aleskandarany MA, Al-kaabi MM, Sonbul SN, Diez-Rodriguez M, Nolan CC, Caldas C, Ellis IO, Rakha EA, Green AR: Ki67 expression in invasive breast cancer: the use of tissue microarrays compared with whole tissue sections. Breast Cancer Res Treat 2017, 164(2):341-348.

18. Sobecki M, Mrouj K, Colinge J, Gerbe F, Jay P, Krasinska L, Dulic V, Fisher D: Cell-Cycle Regulation Accounts for Variability in Ki-67 Expression Levels. Cancer Res 2017, 77(10):2722-2734. 
19. Sinn H-P, Schneeweiss A, Keller M, Schlombs K, Laible M, Seitz J, Lakis S, Veltrup E, Altevogt P, Eidt S et al: Comparison of immunohistochemistry with PCR for assessment of ER, PR, and Ki-67 and prediction of pathological complete response in breast cancer. BMC Cancer 2017, 17(1):124.

20. Batra A: Investigating the regulation of e-cadherin mRNA and protein expression by integrin a3 $\beta 1$ in breast cancer cells. J Clin Onco/2014, 32(15_suppl):1104-1104.

21. Pascal LE, True LD, Campbell DS, Deutsch EW, Risk M, Coleman IM, Eichner LJ, Nelson PS, Liu AY: Correlation of mRNA and protein levels: cell type-specific gene expression of cluster designation antigens in the prostate. BMC Genomics 2008, 9:246.

22. Pallegar N, Ayre DC, Christian S: Repression of CD24 surface protein expression by oncogenic Ras is relieved by inhibition of Raf but not MEK or PI3K. Front Cell Dev Biol 2015, 3:47.

23. Gould VE, Wiedenmann B, Lee I, Schwechheimer K, Dockhorn-Dworniczak B, Radosevich JA, Moll R, Franke WW: Synaptophysin expression in neuroendocrine neoplasms as determined by immunocytochemistry. Am J Patho 1987, 126(2):243-257.

24. Reid MD, Bagci P, Ohike N, Saka B, Erbarut Seven I, Dursun N, Balci S, Gucer H, Jang KT, Tajiri T et al: Calculation of the Ki67 index in pancreatic neuroendocrine tumors: a comparative analysis of four counting methodologies. Mod Pathol 2015, 28(5):686-694.

25. Taylor CR: Predictive biomarkers and companion diagnostics. The future of immunohistochemistry: "in situ proteomics," or just a "stain"? Appl Immunohistochem Mol Morphol 2014, 22(8):555-561.

\section{Figures}
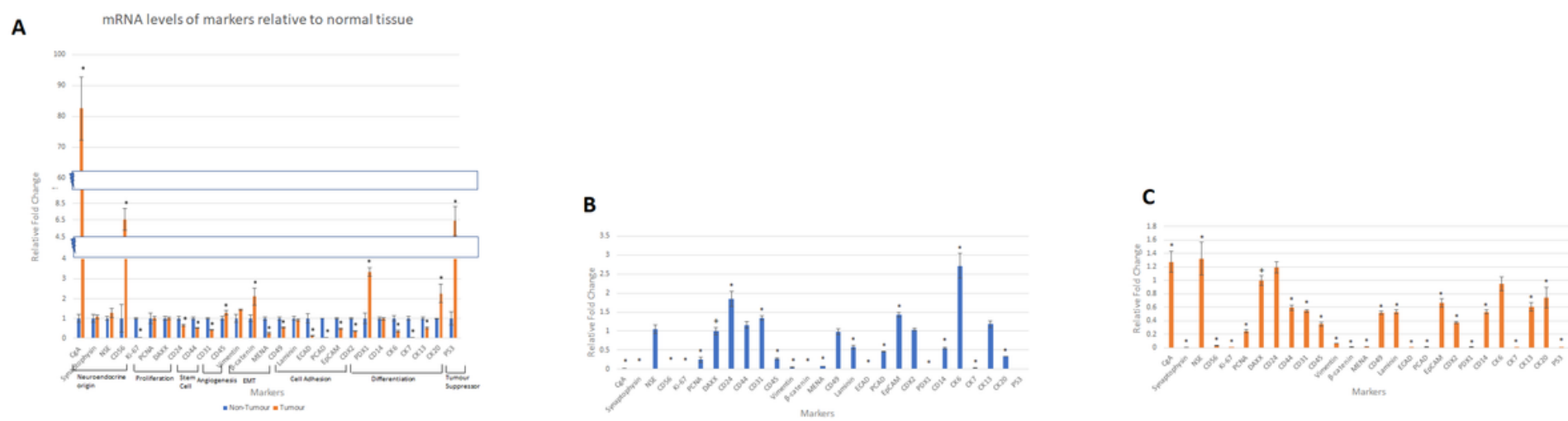

Figure 1

Relative fold change in marker mRNA expression levels using qRT-PCR. * represents the markers that were significantly different in tumour relative to normal. (A) mRNA levels of markers in tumour tissue relative to non-tumour tissue; (B) mRNA levels of markers in non-tumour tissue relative to DAXX; (C) mRNA levels of markers in tumour tissue relative to DAXX. * Represents the markers that were significantly different relative to DAXX expression. + Represents DAXX, used as a control. 
A
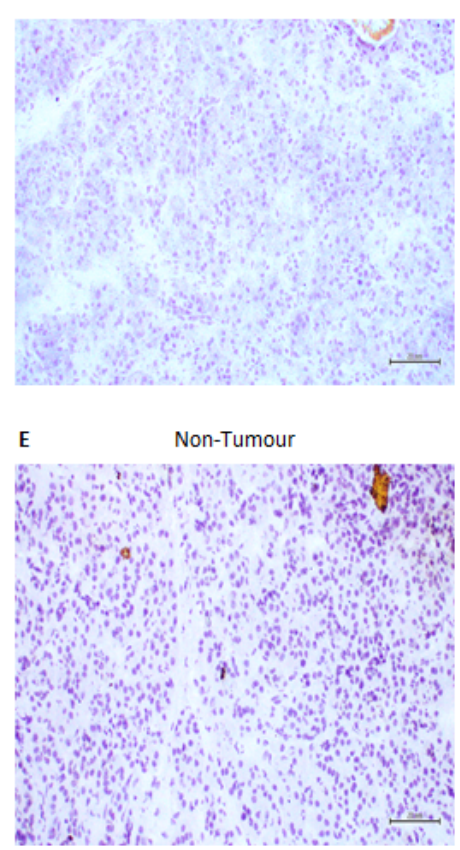

B

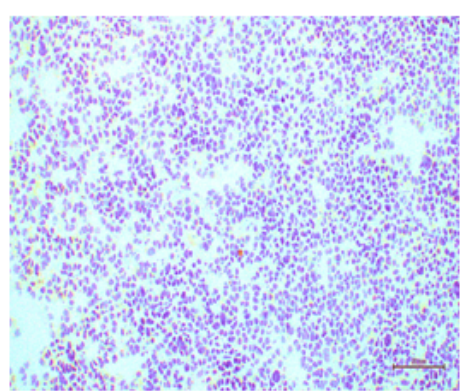

F

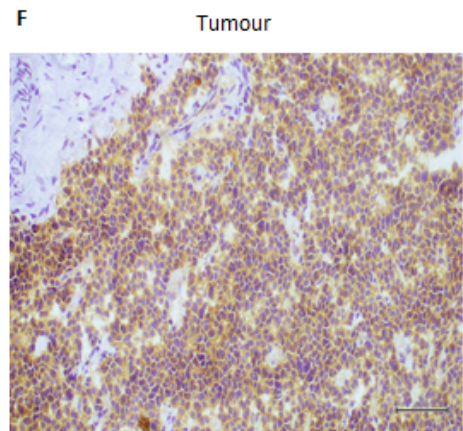

C Non-Tumour
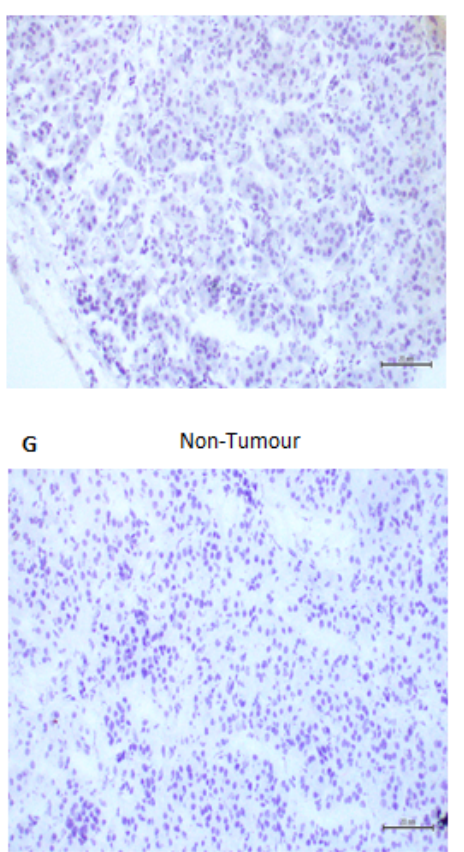
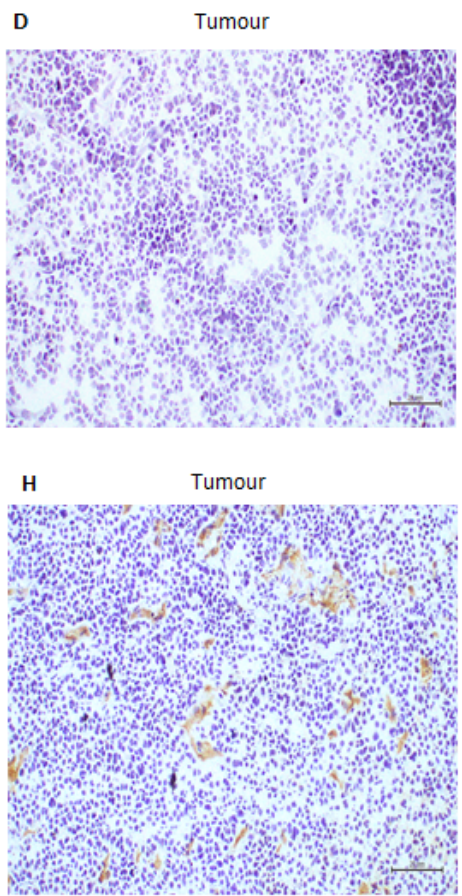

Figure 2

Immunohistochemistry images showing expression levels of CgA (A,B), PCNA (C,D), CD24 (E,F) and CD31 $(\mathrm{G}, \mathrm{H})$ in pancreatic non-tumour and tumour tissue sections.
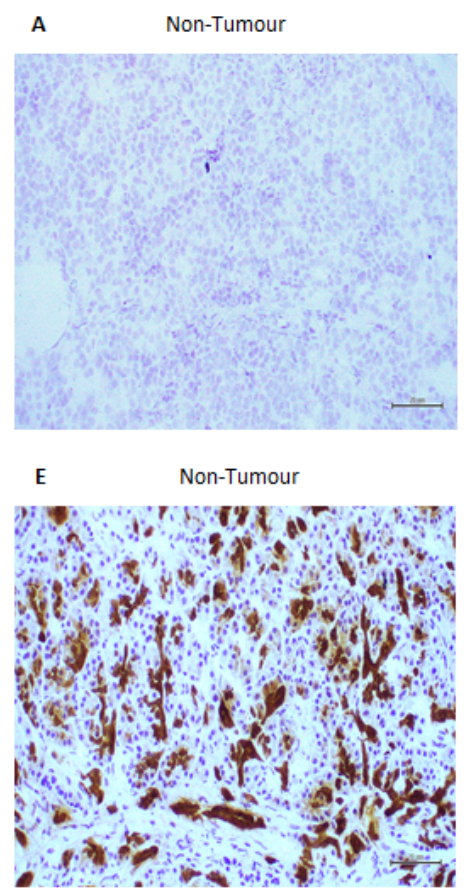

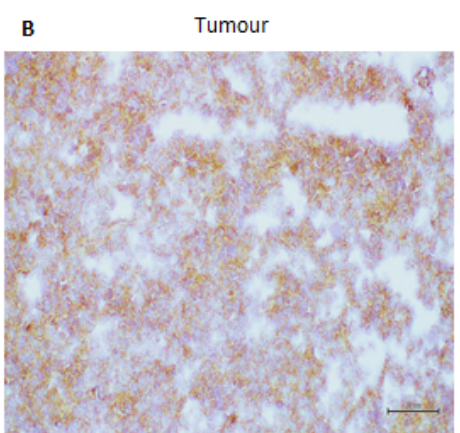

F

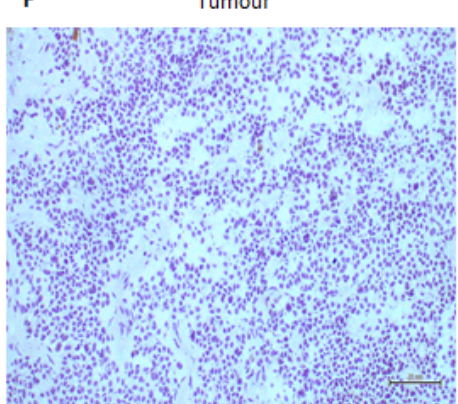

C Non-Tumour

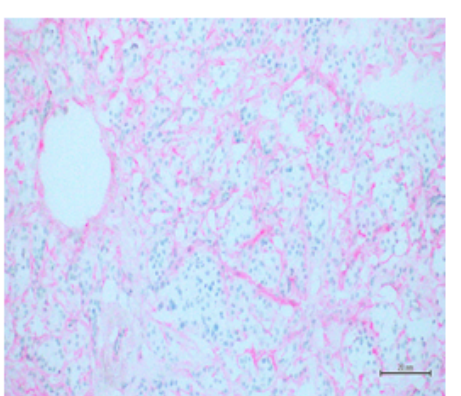

G

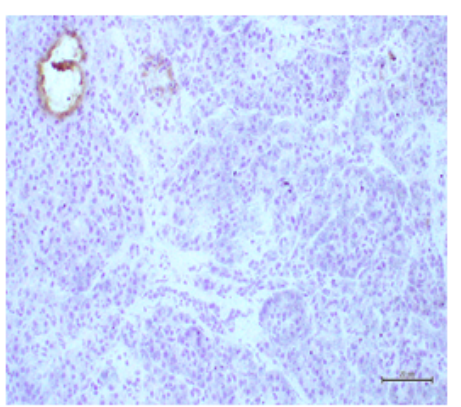

D

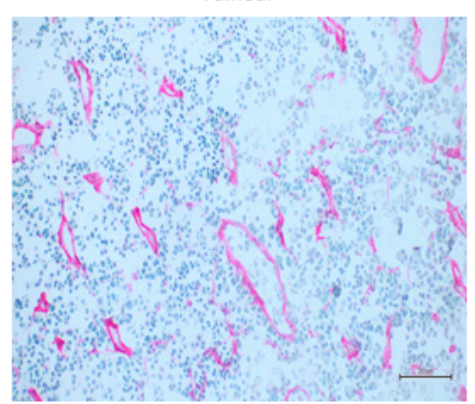

H

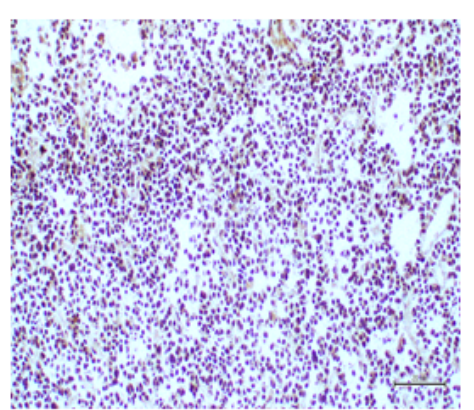

Figure 3

Immunohistochemistry images showing expression levels of $\beta$ - catenin $(A, B)$, Laminin $(C, D), C K 7(E, F)$ and $\mathrm{P} 53(\mathrm{G}, \mathrm{H})$ in pancreatic non-tumour and tumour tissue sections. 